\title{
New Cell Lines from Heliothis virescens: Characterization and Susceptibility to Baculoviruses
}

\author{
Dwight E. Lynn and Martin Shapiro \\ Insect Biocontrol Laboratory, USDA/ ARS, BARC-West, Building 011A, Room 214, Beltsville, Maryland 20705-2350
}

Received December 1, 1997; accepted April 20, 1998

\begin{abstract}
New cell lines from embryos of Heliothis virescens were recently developed. Six primary cultures were initiated in J une 1995. From these initial cultures, two produced sufficient cell growth to allow subcultivation and eventually led to the establishment of seven cell strains, three of which are maintained at low temperatures $\left(17^{\circ} \mathrm{C}\right)$. The strains were compared with a previously established cell line from $\mathrm{H}$. virescens by isozyme analysis and shown to be from the same species. All the strains were inoculated with various baculoviruses, including Autographa californica nucleopolyhedrovirus (NPV), Anagrapha falcifera NPV, Anticarsa gemmatalis NPV, Rachopl usia ou NPV, Lymantria dispar NPV (LdMNPV), Orgyia pseudotsugata NPV (OpSNPV), 0. leucostigma NPV (OIMNPV), and Heli coverpa zea NPV (HzSNPV). All seven strains were highly susceptible to the noctuid NPVs, and large numbers of occlusion bodies (OBs) were produced in most of the inoculated cells. The HzSNPV infection developed at a slower rate (requiring 1 week or more before a substantial number of cells contained OBs compared with 2-3 days for the other three noctuid NPVs). Three of the H. virescens strains were also suscepti ble to OpSNPV although only $10-20 \%$ of the cells produced OBs with this virus. We did not observe cytopathology (CPE) in any cells inoculated with OIMNPV or LdMNPV. Our results suggest that these new strains can be useful for the study and possibly the production of baculoviruses for which no effective cell systems are available and for comparative studies on multiple virus species.
\end{abstract}

Key Words: Heliothis virescens cell lines; Baculoviridae; Autographa californica nucleopolyhedrovirus; Anagrapha falcifera nucleopolyhedrovirus; Anticarsa gemmatalis nucleopolyhedrovirus; Rachopl usi a ou nucleopolyhedrovirus; Orgyia pseudotsugata nucleopolyhedrovirus; and Helicoverpa zea nucleopolyhedrovirus.

\section{INTRODUCTION}

The tobacco budworm, Heli othis virescens, is part of a complex of related species in the Heliothis/ Helicoverpa genera that are very serious pests of cotton, corn, tomato, and tobacco. This insect quickly devel ops resistance to chemical pesticides and is a prime target for biological controls, including insect viruses (Ignoffo, 1966). While other cell lines have been established from this insect (Mclntosh and Ignoffo, 1983; Lynn et al., 1988), the yields of baculovirus occlusion bodies (OBs) in these cells were lower than can be obtained in other lepidopteran lines (Mcl ntosh et al., 1985). All of these lines were established from larval or pupal reproductive tissue. In previous studies with cell lines from the gypsy moth, we have shown other cell sources for established cell lines, including embryos, can provide better substrates for the replication of nucleopolyhedrovirus (NPV) (Lynn et al., 1989).

In this study, we used $\mathrm{H}$. virescens embryos to initiate cultures. Of six initial primary cultures, two showed sufficient cell grow to enable subculturing and led to the development of cell lines. Seven different strains were established as a result of various selection and growth conditions. These strains were characterized by morphology and by isozyme analyses. They were subsequently screened for replication of various NPVs and shown to be susceptible to Autographa cal ifornica NPV (AcM NPV), Anagrapha fal cifera NPV (AfMNPV), Anticarsa gemmatalis NPV (AgMNPV), Rachoplusia ou NPV (RoMNPV), Orgyia pseudotsugata NPV (OpSNPV), and Helicoverpa zea NPV (HzSNPV). These new lines should be useful in the study and production of baculoviruses, especially HzSNPV for which no satisfactory system is currently available.

\section{METHODS AND MATERIALS}

Cell line devel opment. Primary cultures were isolated from middevel opment $\mathrm{H}$. virescens eggs obtained from Dr. J ames F oster (Dupont Ag, Newark, DE ) using procedures described in Lynn (1996). Six primary cultures were initiated in BML-TC/10 medium (TC-100 from Sigma Chemical Co., St. Louis, MO) modified as previously described (Lynn et al., 1988) in J une, 1995 and maintained at $26^{\circ} \mathrm{C}$. Medium was added or re- 
placed on these cultures at 7- to 14-day intervals until enough cells were available for subculturing. Of the initial six cultures, two developed into cell lines (initially designated I PLB-H VE 1 and I PLB-HvE 6).

During early passages, the H vE 6 line showed heterogeneous morphologies between the attached and suspended cell types. Two separate strains were isolated from this culture by selecting for attachment and nonattachment, respectively, and were designated as IPLB-HvE6a and IPLB-HvE6s. The attached strain was selected by firmly striking the culture prior to replacing the medium at weekly intervals (or at the time of subcultivation when cell growth was sufficient to actually split the cultures). For the suspended strain, cultures were treated gently to avoid disturbing attached cells when transferring cells to a new culture. After 9 months in culture (4 to 15 passages), we began to maintain separate cultures of the three strains at $17^{\circ} \mathrm{C}$ in addition to the $26^{\circ} \mathrm{C}$ standard temperature (these low temperature strains were designated IPLBH vE 1-It, I PLB-HvE6a-It, and IPLB-HvE6s-It). An additional suspended substrain was isolated from $\mathrm{HvE} 1$ (now designated I PLB-H vE Ia and I PLB-H vE Is) resulting in a total of seven strains.

Maintenance Because of differences in growth characteristics between the attached and suspended strains, different cell passage procedures are necessary. The four attached strains (HvE la, HvE 1-It, HvE 6a, and HvE6a-It) were subcultured using trypsinization. The medium was decanted from a confluent $25-\mathrm{cm}^{2}$ culture and the cell monolayer was rinsed with $2.0 \mathrm{ml}$ trypsinization buffer (Ca- and Mg-free Hanks' phosphate-buffered saline; Lynn, 1996). The rinse was discarded and an additional $1.0 \mathrm{ml}$ of buffer containing 50 $\mu \mathrm{g} / \mathrm{ml}$ VMF trypsin (Worthington Enzymes, Freehold, NJ ) was added. After 5 min at room temperature, the trypsin solution was decanted and the culture held for an additional 5-10 min. Fresh TC-100 medium was then added and thecell suspension was distributed to new cultures.

For the suspended cell strains (HvE 1s, HvE6s, and HvE6s-(t), medium from a mature culture was simply transferred to fresh TC-100 medium in a new flask. If the split ratio was less than 1:4, the cell suspension from the mature culture was centrifuged ( 5 min at $50 \mathrm{~g}$ ) and resuspended in fresh medium beforetransfer to the new culture flask.

Since these cultures were initiated, the cells have been maintained in Falcon, Nunclon, or Greiner culture flasks with no discernible difference in growth. In the early stages of establishment, all cultures were split at 1:2 after cultures reached sufficient density as judged by microscopic examination. The split ratios were altered as the cell growth rate increased during adaptation to the culture conditions. The $26^{\circ} \mathrm{C}$ cultures are currently subcultured on a 1-week schedule and the $17^{\circ} \mathrm{C}$ cultures are subcultured at 2-week intervals.
Characterization. Extracts of the cells from the seven strains were prepared using the Authentikit isozyme system. The extracts were applied to $1 \%$ agarose gels, electrophoresed at $160 \mathrm{~V}$ for $25 \mathrm{~min}$, and then stained for malic enzyme (ME, EC 1.1.1.40), isocitrate dehydrogenase (ICD, EC 1.1.1.42), phosphoglucose isomerase (PGI, EC 5.3.1.9), or phosphoglucomutase (PGM, EC 2.7.5.1). The stains, buffers, and gels were obtained from I nnovative Chemistry (Marshfield, MA). An extract from IPLB-HvT1 cells (Lynn et al., 1988) was included on the gels for comparison and the isozyme migration patterns were also compared with other cells maintained in our laboratory.

Virus studies. The viruses tested in these studies include AcMNPV, AfMNPV, AgMNPV, RoMNPV, OpSNPV, O. leucostigma NPV (OIMNPV), Lymantria dispar NPV (LdMNPV), and HzSNPV. The virus inocula were all derived from cell cultures that are permissive for the respective viruses. The source of viruses was described previously, Lynn and Shapiro (1997), except for HzSNPV. This virus was prepared by per os infection of third instar $\mathrm{H}$. zea larvae on an artificial diet (Bell et al., 1981). Infected larvae were surface disinfected by immersion in $70 \%$ ethanol for 10 min, air dried, and then bled through a cut proleg into cold TC-100 medium (on ice) supplemented with 50 $\mu \mathrm{g} / \mathrm{ml}$ gentamicin sulfate (Sigma) and $80 \mu \mathrm{g} / \mathrm{ml}$ reduced glutathione(Sigma). One milliliter of medium was used per larvae (infected larvae were approximately 2.5-3 $\mathrm{cm}$ long). The hemolymph-containing medium was centrifuged (15 min at $350 \mathrm{~g}$ ) and the supernatant was passed through a $0.45 \mu \mathrm{m}$ Uniflo filter. This filtrate was used to initiate infection of IPLB-HVT1 cells and supernatants from infected cultures were used for subsequent tests.

To screen for virus susceptibility, cells of each strain were transferred to 24-well tissue culture plates (Costar) in $1.0 \mathrm{ml}$ TC-100 medium supplemented with 50 $\mu \mathrm{g} / \mathrm{ml}$ gentamicin sulfate. Ten to $100 \mu \mathrm{l}$ of each virus inocula was added to a well of each cell strain. Due to the growth characteristics of some of the $\mathrm{H}$. virescens cell strains, accurate cell counts were not possible. However, based on microscopic examination of the cultures, we estimated that the virus inocula used in these studies represent a multiplicity of infection of 0.1-10 infectious particles per cell (as determined by endpoint assay of each virus in an appropriate cell line). In our experience, this level of inoculation should be adequate for determining susceptibility of these cells to the various viruses. The inoculated plates were sealed with masking tape and maintained at room temperature (approximately $22^{\circ} \mathrm{C}$ ) and examined with an inverted phase contrast Leitz microscope at intervals up to 1 month for the presence of viral OBs.

A quantitative study was performed on the new strains with HzSNPV since no good cell systems exist for its study or production. Cells were inoculated into 

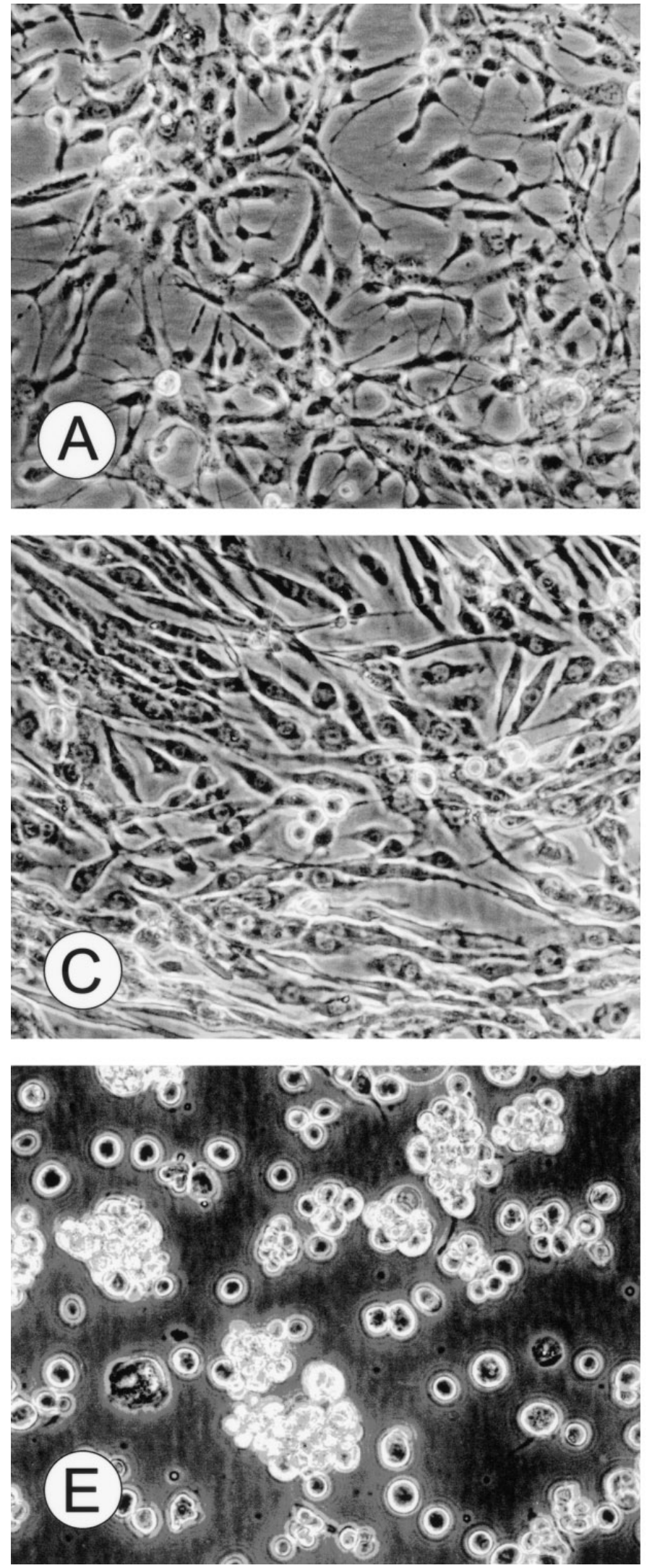

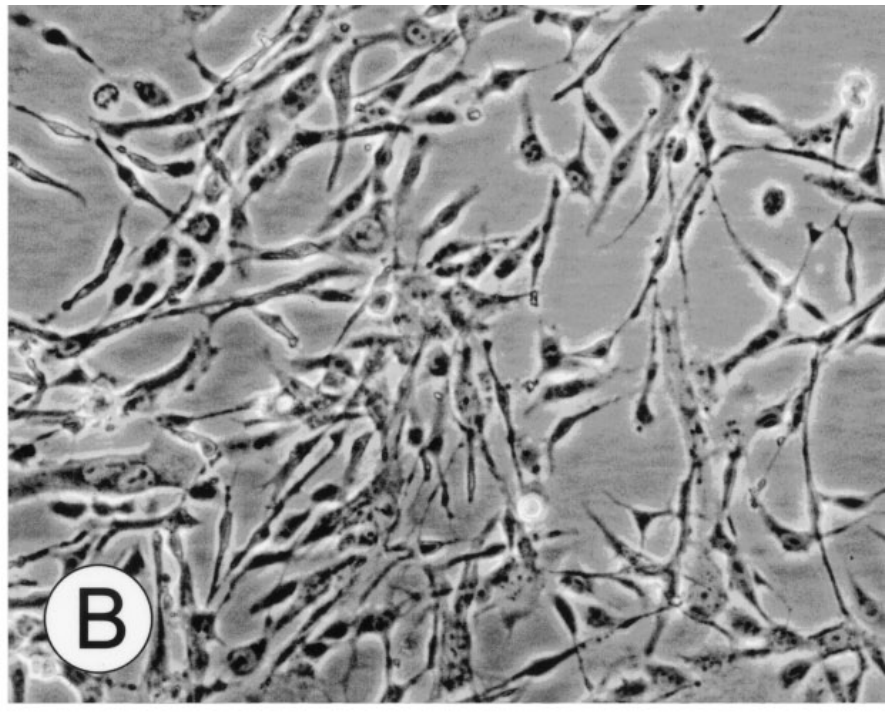

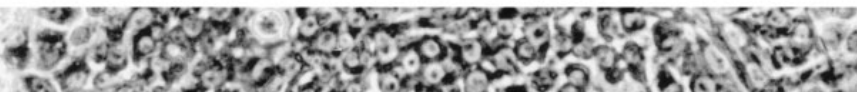

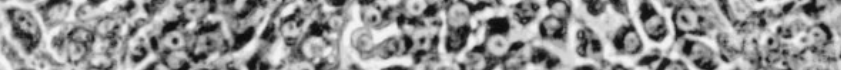

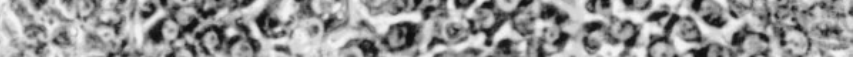

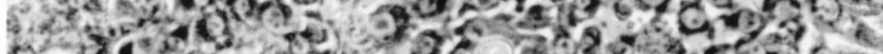

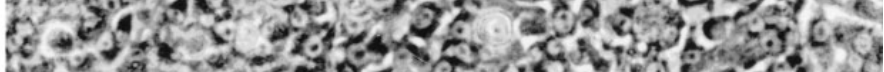

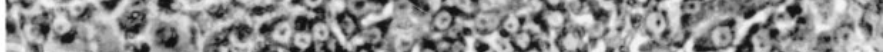
I.

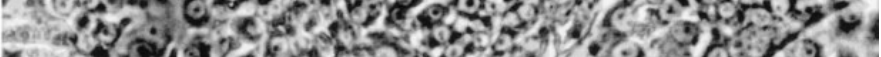

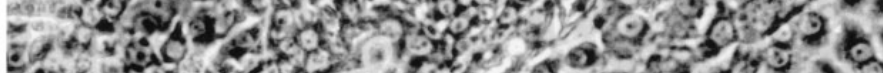

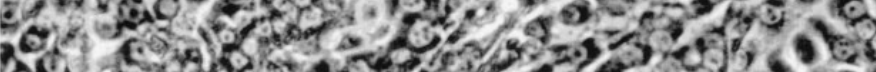

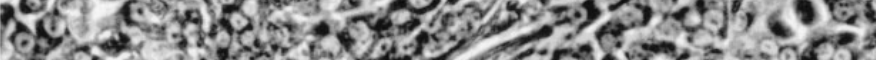

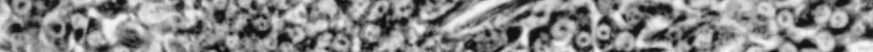

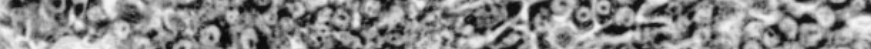

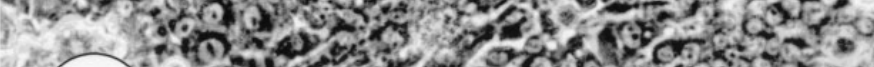

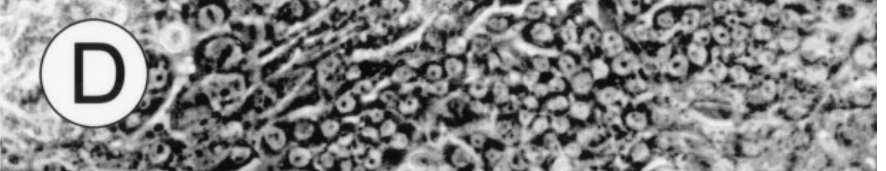

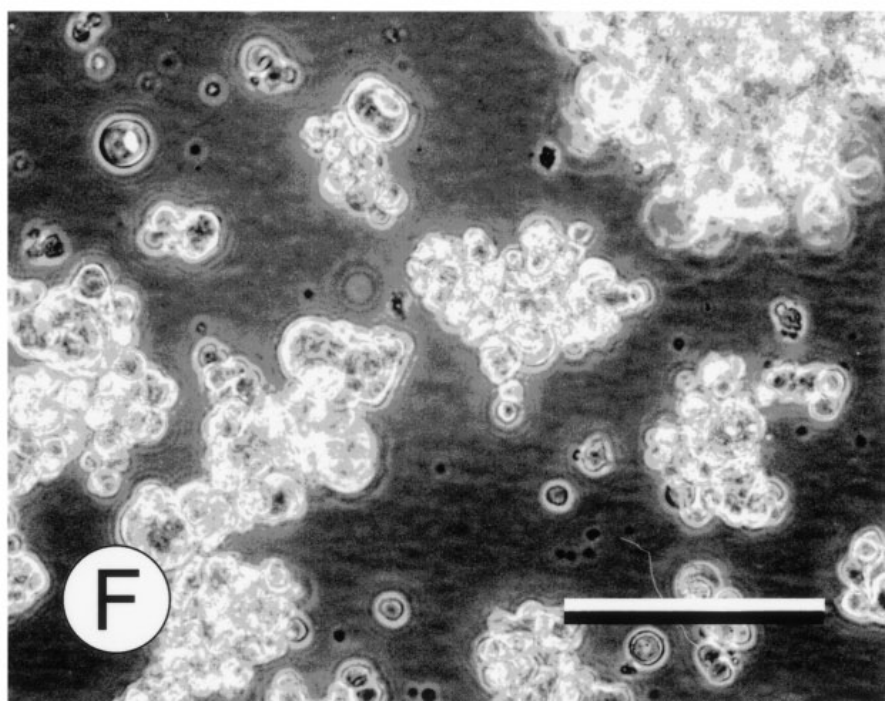

FIG. 1. . Phase contrast micrographs of new H. virescens cell strains. (A) IPLB-HvE la; (B) IPLB-HvE 1-It; (C) IPLB-HvE6a; (D) IPLB-H vE6a-It; (E) IPLB-HvE-1s; (F) IPLB-HvE6s. All figures are at the same magnification. Bar, $100 \mu \mathrm{m}$. 
24-well culture plates as above. Cultures were initiated at three densities equivalent to $0.5,1.0$, and $2.0 \times$ the density routinely used to initiate cultures during normal cell passage (again, this method was used because of the inability to make accurate counts of the aggregated cells in some of the cultures). The cells were inoculated with $10 \mu \mathrm{l} \mathrm{HzSNPV}$ from previously infected cell cultures (two passages in H vE 6a cells). Plates were sealed with masking tape and maintained at room temperature for 9 days. The medium was replaced with $1 \mathrm{ml}$ deionized water and $40 \mu \mathrm{l} 10 \%(\mathrm{w} / \mathrm{v})$ sodium laurel sulfate (Sigma) was added. Repeated pipetting through a glass Pasteur pipette (Corning Glass Works, Corning, NY) was used to disrupt the cells. The plates were set aside for 1-3 days to allow the OBs to settle, after which an estimate of OBs was determined by counting a specific surface area in each well as measured by a photographic ocular in a Leitz inverted phasecontrast microscope.

\section{RESULTS AND DISCUSSION}

Seven cell strains have been established from embryos of the tobacco budworm, $\mathrm{H}$. virescens. The morphologies of these cells are seen in Fig. 1. HvE la, HvE 1-It, and HvE 6a are each predominantly fibroblast like, firmly attached cells although the HvEla and H vE 1-It cells are narrow and form a web-like network (Figs. 1A and 1B) while the HvE6a cells are broader and are closely aligned to each other in parallel arrays (Fig. 1C). Twenty to $30 \%$ of HvE6a-lt are similar to HvE 6a but at least $70 \%$ of these cells exhibit epitheliallike morphology (the ratio of the two cell types varies somewhat with the age of the culture) (Fig. 1D). HvE Is cells are predominantly round and suspended and occur singly or in small clumps (Fig. IE), while both HvE $6 s$ and H vE 6s-It are large aggregates of suspended cells with somecells occurring as well organized vesicles (Fig. IF). The low temperature strains were selected because cell lines may develop with a broader host range to various insect viruses at low temperature (Winstanley and Crook ,1988).

The similarity of the isozyme patterns of these cells to HVT1, another $H$. virescens line maintained in our $\mathrm{lab}$, indicates that they are derived from $\mathrm{H}$. virescens (Fig. 2). Of the four systems used, the migration of ME and PGI enzymes was identical among all seven new strains and HVT1. One of two allozymes present in the PGM gels differed between the new strains and HVT1, possibly due to differences in the source of the originating insects or the different tissue source of the cells. In addition to the migration of this allozyme differing slightly between the new strains and HvT1, it was also much less intense with the three HvE 1 strains. I nter estingly, noICD enzyme activity was detected in any of the low-temperature strains. The PGM isozyme patterns of the new strains were similar to patterns obtained with several Trichoplusia ni cell lines (data not shown) but

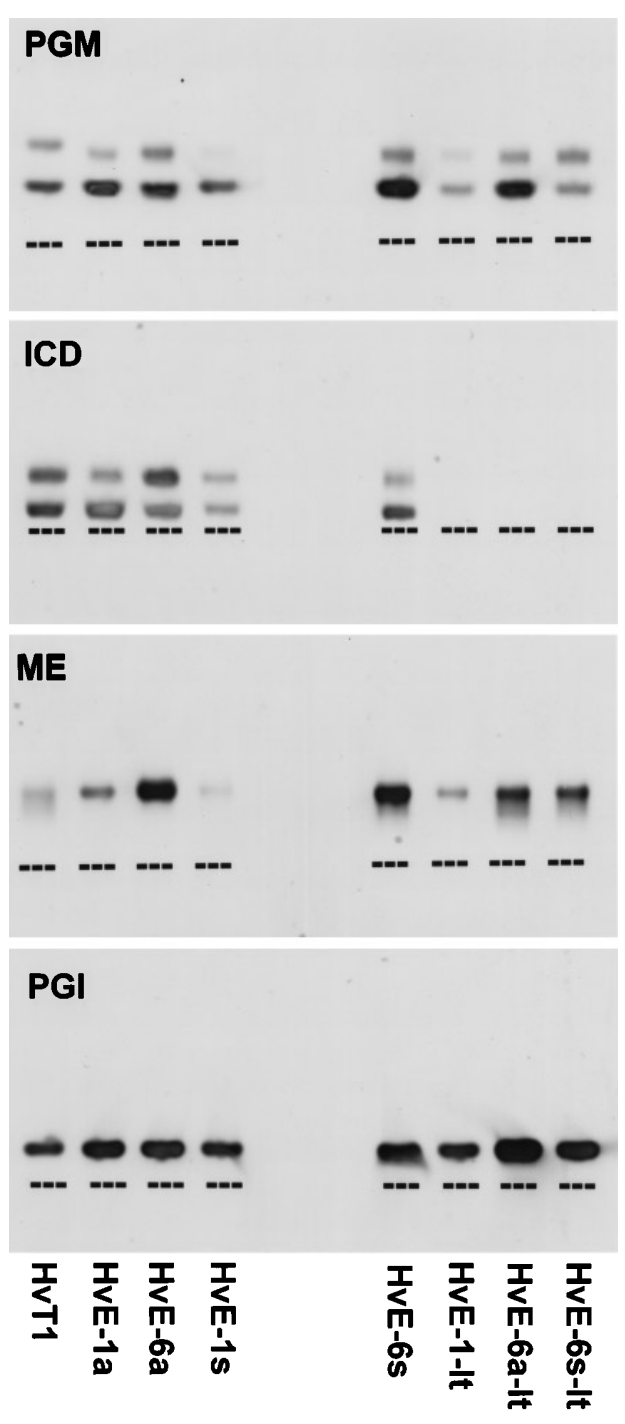

FIG. 2. Electrophoresis patterns on gels stained for phosphoglucomutase (PGM), isocitrate dehydrogenase (ICD), malic enzyme $(\mathrm{ME})$, and phosphoglucose isomerase (PGI). All materials were from theAuthentikit system. The triple dash mark (- - -) shows the location where the individual cell extracts (from the cell line indicated at the bottom of the figure) were applied to the gels. Samples were electrophoresed 25 min prior to staining with the indicated substrate.

none of the other enzymes matched these or any other lines maintained in our laboratory. Based on the isozyme results, we are confident that the strains described in this paper are unique.

All of the new strains were highly susceptible to the five noctuid viruses tested (ACMNPV, AfMNPV, AgMNPV, HzSNPV, and RoMNPV) while being only slightly susceptible to OpSN PV and showing no cytopathology with OIMNPV or LdMNPV (Table 1). Of these, the replication of AgMNPV was most surprising. With the exception of cells from the same species (UFLAG286, Sieburth and Maruniak, 1988), it replicates quite poorly in most of the cell lines maintained in our 
TABLE 1

Replication of Various Nudeopolyhedroviruses in New Cell Strains from $H$. virescens

Cell lines

\begin{tabular}{lcccccccc}
\cline { 2 - 7 } Virus & \multicolumn{9}{c}{ HvEla } & HvEls & It & HvE6a & It & HvE6s & It \\
\hline HzSNPV & +++ & $(+)$ & +++ & +++ & +++ & +++ & +++ \\
ACMNPV & ++ & ++ & ++ & +++ & ++ & +++ & ++ \\
AfMNPV & +++ & +++ & ++ & +++ & +++ & ++ & ++ \\
RoMNPV & ++ & +++ & ++ & +++ & +++ & ++ & ++ \\
LdMNPV & - & nt & - & - & - & - & - \\
OIMNPV & - & - & - & - & - & - & - \\
OpMNPV & - & $\mathrm{nt}$ & + & + & + & - & - \\
AgMNPV & +++ & +++ & +++ & +++ & +++ & +++ & +++
\end{tabular}

Note +++ , over $90 \%$ of cells with CPE (OBs);,$++ 50-80 \% ;+$, 10-20\%. nt, not tested. - , no CPE apparent.

laboratory but produces as many OBs in at least two of the new strains as UFL-AG286 (unpublished preliminary data).

The quantitative studies with HzSNPV revealed that several of the new strains were capable of producing more OBs than the previously available HVT1 line (Fig. 3). As mentioned earlier, the clumping of several of these strains makes counting these cells difficult. For this reason, the production of OBs is presented on a per milliliter basis. In assaying the various strains for HzSNPV production, three cell densities (representing $0.5,1$, and $2 \times$ the normal number used to initiate cultures) were tested with a constant amount of virus. In these experiments, the two attached, $26^{\circ} \mathrm{C}$ strains, HvE $1 a$ and HvE6a, produced the most OBs. As with other cell lines that we have compared (Lynn et al.,

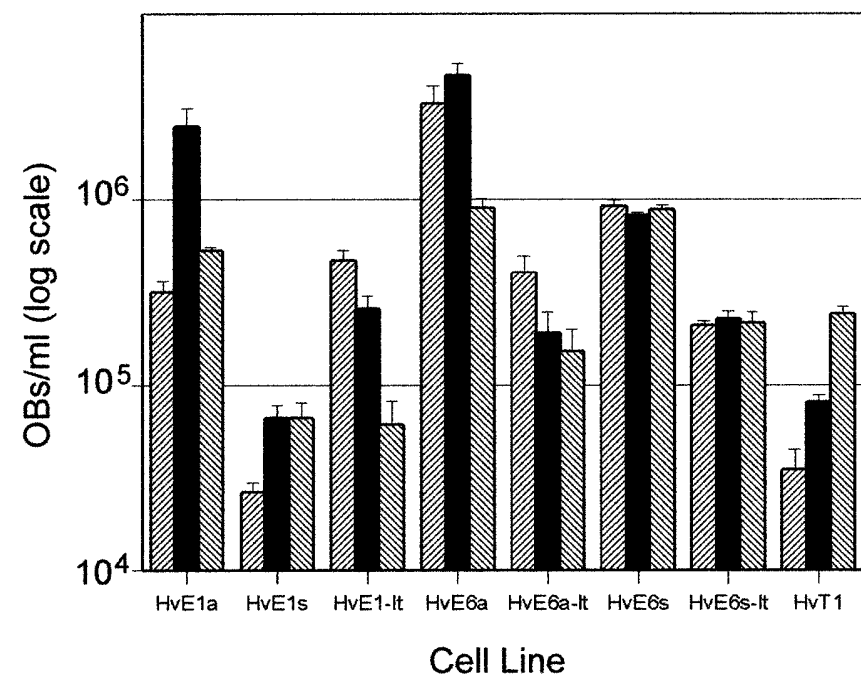

FIG. 3. Production of HzSNPV occlusion bodies (OBs) in new cell strains. The first bar in each series is from cells that were set up at half the normal density, the middle bar (solid black) was set up at the normal density, and the third bar was set up at twice the normal density. The number of OBs was counted by lysing cells 9 days postinfection. Each bar is the mean of three replicate experiments.
1989; Lynn and Shapiro, 1997), there is no good explanation as to why different cell lines produce variable amounts of virus. We suspect that this ultimately relates to the actual cell type represented by the cultured cells since it is clear that different tissues in the insect become infected at different times and produce different quantities of virus. We are currently devel oping an assay for the nonoccluded virus so we can determine if there are also quantitative differences with that form of the virus. These new strains appear to have good potential for studying HzMNPV and, with some further optimization of the growth conditions, they may also be useful in a production scheme for this virus. Weare also currently examining the potential of these cells for replicating a granulosis from Helicoverpa armigera.

\section{ACKNOWLEDGMENTS}

We thank Ms. Vallie Bray for preparation of media and supplies for the cell culture research and Ms. Marla Hunt and Gail Rosenberry for maintenance of insects used in preparing the virus samples. Mention of proprietary or brand names is necessary to report factually on the available data; however, the USDA neither guarantees nor warrants the standard of the product, and the use of the name by the USDA implies no approval of the product to the exclusion of others that may also be suitable.

\section{REFERENCES}

Bell, R. A., Owens, C. D., Shapiro, M., and Tardif, J . G. R. 1981. Development of mass rearing technology. In "The Gypsy Moth: Research towards I ntegrated Pest Management" (C. C. Doane, and M. L. McManus, Eds.), pp. 599-633. U. S. Department of Agriculture Technical Bulletin No. 1584.

I gnoffo, C. M. 1966. Susceptibility of the first-instar of the bollworm, Heliothis zea, and the tobacco budworm, Heliothis virescens, to Heliothis nuclear-polyhedrosis virus. J . I nvertebr. Pathol. 8, 531536.

Lynn, D. E. 1996. Development and characterization of insect cell lines. Cytotechnology 20, 3-11.

Lynn, D. E., Dougherty, E. M., McClintock, J . T., and Loeb, M. 1988. Development of cell lines from various tissues of Lepidoptera. In "Invertebrate and Fish Tissue Culture" (Y. Kuroda, E. Kurstak, and K. Maramorosch, Eds.), pp. 239-242. J apan Scientific Societies Press, Tokyo.

Lynn, D. E., Dougherty, E. M., McClintock, J. T., and Shapiro, M. 1989. Comparative replication of Lymantria dispar nuclear polyhe drosis virus strains in three continuous-culture cell lines. Appl. Environ. Microbiol. 55, 1049-1051.

Lynn, D. E., and Shapiro, M. 1997. Virus susceptibilities of new cell lines from embryos of the whitemarked tussock moth. In Vitro Cell. Dev. Biol. Anim. 33, 487-488.

Mclntosh, A. H., and I gnoffo, C. M. 1983. Characterization of five cell lines established from species of Heliothis. Appl. Entomol. Zool. 18, 262-269.

Mcl ntosh, A. H., I gnoffo, C. M., and Andrews, P. L. 1985. In vitro host range of five baculoviruses in lepidopteran cell lines. Intervirology 23, 150-156.

Sieburth, P. J ., and Maruniak, J . E. 1988. Growth characteristics of a continuous cell line from the velvetbean caterpillar, Anticarsa gemmatalis Hübner (Lepidoptera: Noctuidae). In Vitro Cell. Dev. Biol. 24, 195-198.

Winstanley, D., and Crook, N. E. 1993. Replication of Cydia pomonela granulosis virus in cell cultures. J . Gen. Virol. 74, 1599-1609. 